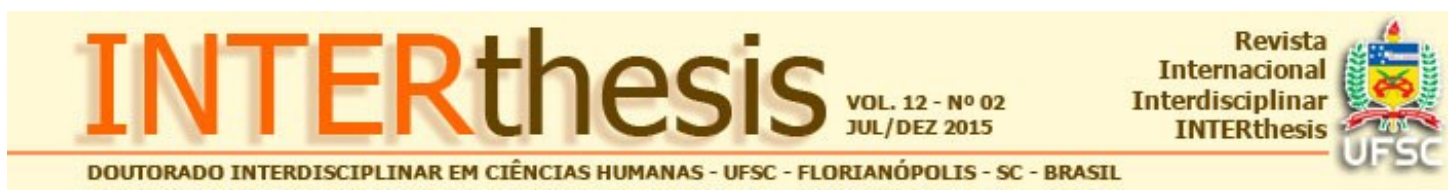

\title{
CIÊNCIA DA INFORMAÇÃO E APTIDÃO AGRÍCOLA: ABORDAGENS INTERESCALARES PARA PLANEJAMENTO DE USO DA TERRA
}

\author{
Vitor Vieira Vasconcelos ${ }^{1}$ \\ Paulo Pereira Martins Junior ${ }^{2}$
}

\section{Resumo:}

As inovações técnicas e metodológicas de programação, compartilhamento em rede, análise de sistema de informação e sistemas de informação geográfica (SIG) permitem a representação, armazenamento, tratamento e disseminação de informações que antes não se havia disponíveis para diversos atores interessados. Partindo desse contexto, apresenta-se proposta de modelagem conceitual sobre o uso integrado das metodologias de Aptidão Agrícola para o desenvolvimento de sistemas de auxílio à decisão sobre uso da terra, auferidas por meio de metodologias da Ciência da Informação. São estruturados diagramas de modelagem de conhecimento por meio dos métodos CommonKADS e Unified Modeling Language (UML), para simular o processamento de perguntas básicas de um produtor rural, tais como "O que plantar?", "Onde plantar?", "Como plantar?". Por fim, é apresentado uma matriz interdisciplinar explicitando a relação entre a aptidão Agrícola por cultivar com atributos de solo e relevo, para auxílio à decisão em escala de detalhe. O sistema proposto afigura-se um eficaz instrumento de orientação e educação agropecuária e ambiental, por demonstrar alternativas ecologicamente sustentáveis ao usuário e explicar a justificativa econômica para tais propostas.

Palavras-chave: Agronomia. Sistemas de Informação Geográfica. Ciência da Informação. Sistemas de Auxílio à Decisão. Meio Ambiente.

\section{INTRODUÇÃO}

O planejamento de uso da terra para atividades agro-silvo-pastoris tem se beneficiado, a longos anos, das técnicas de mapeamento para aptidão de atividades econômicas rurais. Entre as técnicas utilizadas tradicionalmente, encontram-se a Avaliação de Capacidade de Uso do Solo (LEPSCH et al., 1991), e o Sistema

\footnotetext{
${ }^{1}$ Pós-doutor no Stockholm Environment Institute, Asia Centre, Bangkok, Tailândia - Bolsista CNPq. Doutor em Ciências Naturais pela Universidade de Ouro Preto, Ouro Preto, MG, Brasil, com doutorado-sanduíche em Engenharia de Recursos Hídricos na Universidade de Chulalongkorn, Tailândia. E-mail: vitor.v.v@gmail.com

2 Doutor em Ciências da Terra pela Université Pierre et Marie Curie, Lise, França. Professor no Departamento de Geologia, Escola de Minas, Universidade Federal do Ouro Preto, na Rede de Ciência dos Materiais, e no mestrado em Sustentabilidade Sócio Ambiental e Econômica da Escola de Minas, Ouro Preto, MG. Pesquisador do IGTEC - Instituto de Geoinformação e Tecnologia, Belo Horizonte, MG, Brasil. E-mail: maerteyn@gmail.com
}

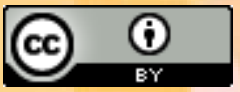
Esta obra foi licenciada com uma Licença Creative Commons - Atribuição 3.0 Não Adaptada. 
FAO/Brasileiro de Avaliação de Aptidão Agrícola das Terras (RAMALHO FILHO e BEEK, 1995). Ambos fornecem uma indicação preliminar sobre a aptidão das terras para diferentes atividades produtivas, sobre sistemas de manejo diferenciados.

Também já tradicional no meio agrícola brasileiro é o Zoneamento Agroclimatológico (ou agroclimático) por cultivares. Exemplos típicos são os zoneamentos para a Cana de Açúcar (MANZATTO et al., 2009) e da NogueiraMacadâmia (SCHNEIDER et al., 2011), com foco nas possibilidades de expansão agrícola das respectivas cultivares para o Brasil. Na última década, também se destacam como os zoneamentos agrícolas de risco climático desenvolvidos pela Embrapa, com fins de subsidiar o seguro rural (OZAKI, 2007), bem como sua extensão para simular os riscos frente às projeções de mudanças climáticas (ASSAD et al., 2013). É característico dos zoneamentos agroclimatológicos a distribuição espacial das restrições de umidade, precipitação, temperatura, bem como riscos de geadas e outras intempéries (WOLLMANN e GALVANI, 2013).

Mais recentemente, uma nova modalidade de carta também tem se somado à cartografia de planejamento de expansão rural. Trata-se das cartas de Zoneamento Ecológico-Econômico, embora a formatação adequada de suas metodologias ainda seja motivo de discussão acadêmica (MARTINS JUNIOR et al., 2009). Os atributos mapeados englobam, no mínimo, as restrições legais de ocupação territorial (unidades de conservação, áreas de preservação permanente, entre outros). Contudo, também podem refletir acordos sociais de conservação de territórios especiais, como corredores ecológicos e outras áreas de relevante interesse de preservação (BOLFE et al. 2015). Mais ainda, o que embasa o conceito de Zoneamento Ecológico-Econômico é uma tentativa de integrar os aspectos de recursos naturais, sociais e econômicos sobre uma proposta territorial de desenvolvimento sustentável (VASCONCELOS et al., 2013). A amplitude de suas metas é desafiante, especialmente quantos aos limites epistemológicos da cartografia moderna.

\subsection{OBJETIVOS}

Este artigo tem como objetivo abordar como o planejamento do uso da terra pode ser potencializado por meio da abordagem técnica da Ciência da Informação. Argumenta-se que, com os desenvolvimentos tecnológicos de bases de dados, 
programação lógica, sistemas de informação geográfica (SIG) e disseminação por rede, torna-se possível oferecer instrumentos úteis para o desenvolvimento agropecuário, em diferentes escalas de planejamento. A concepção de uma estrutura informacional integrada também abre a possibilidade de reunir vários conhecimentos técnicos e científicos que até o momento encontram-se esparsos em diversos meios de disseminação.

É proposto o esquema conceitual para orientar a elaboração de sistemas especialistas de auxílio à decisão (SEAD) que envolvam a aptidão agrícola. A organização do conhecimento que se propõe visa auxiliar: [1] atender às demandas práticas para projetos executivos em bacia hidrográficas, bem como para medidas mitigadoras e o planejamento de projetos agrícolas, [2] responder a um elenco de questões que normalmente serão feitas por usuários que têm dúvidas sobre o que convém fazer, ou não, em sua propriedade rural dadas as condições geoambientais, [3] reconhecer os problemas em cada propriedade rural, acessando-se assim às bases de dados cartográficos e científicos para que as respostas sejam as mais adequadas com a realidade representável, [4] atender tanto ao proprietário rural quanto ao gestor, que devem desenvolver raciocínio de escala local, a subregional a regional.

\section{METODOLOGIA}

Foi utilizada a metodologia de Engenharia de Conhecimento CommonKads, utilizada para implementação e gerenciamento de sistemas de conhecimento intensivo (FAISAL et al., 2011). Inserido na metodologia CommonKADS, foi empregada a linguagem UML (SEIDL et al., 2015), utilizada em Engenharia de Programação (software) para modelagem padronizada no processo de modelagem de informações e estruturas de inferência.

O processo de elicitação (obtenção) e modelagem de dados e de fluxos de informações foram obtidos em trabalho conjunto entre profissionais especialistas em pedologia e planejamento territorial da Fundação Centro Tecnológico de Minas Gerais - CETEC-MG -, do Instituto de Geociência Aplicadas de Minas Gerais - IGA -, da Universidade Federal de Ouro Preto - UFOP - e da Pontifícia Universidade Católica de Minas Gerais - PUC-MINAS. Os trabalhos foram realizados a partir do seguinte histórico de projetos de pesquisa, com aplicação na bacia do Rio Paracatu: 
- Projeto CRHA - "Conservação de Recursos Hídricos no âmbito da Gestão Ambiental e agrícola de Bacia Hidrográfica" (MARTINS JUNIOR et al., 2006), financiado pelo MCT/Finep - CT-Hidro (2002-2006).

- Projeto ACEE - "Arquitetura de Conhecimentos em Ecologia-Economia" (MARTINS JUNIOR et al., 2007), financiado pelo MCT/CNPq (2005-2007).

- Projeto SACD - "Sistemas de Arquitetura de Conhecimentos e de Auxílio à Decisão na Gestão Geoambiental e Econômica de Bacias Hidrográficas e Propriedades Rurais" (MARTINS JUNIOR et al., 2010), financiado pela Fapemig (2010-2011).

Dentre os outros métodos de aquisição de conhecimentos, foram utilizados: redação de textos, listas de conceitos com as suas definições, mapas, tabelas, hierarquias, fluxogramas, organogramas, esquemas e resolução de casos exemplos (casos clássicos, reais e hipotéticos). Tal variedade se justifica afim de que o especialista se expresse das mais diversas formas possíveis, pois em cada uma o conhecimento apresenta detalhes que não se mostraram nos anteriores. Ressalta-se que o conhecimento apresentado não provém apenas da aquisição por profissionais especialistas, mas também de livros, publicações, tabelas, arquivos, bancos de dados, mapas, bases SIG, e outros. Esses dados também complementam a base de conhecimento do que se propõe como sistema multiespecialista.

Nesse contexto, o trabalho desenvolvido consistiu nas seguintes etapas:

1) Modelagem conceitual dos domínios de conhecimento (visão, organização e inferência), tarefas e comunicação (interface e interação de módulos), de acordo com o método CommonKADS;

2) Diagramas de uso de caso para auxílio à decisão, no padrão UML

3) Modelagem de objetos para estruturação de dados, com diagramas de contexto, classes e atividades em modelos de estrutura UML

4) Sistematização de critérios de aptidão agrícola em uma matriz de conhecimentos interdisciplinares

\section{DESENVOLVIMENTO}

Com a progressiva elaboração de bases de dados cartográficas disponíveis por SIG, tornou-se possível realizar operações de algoritmos lógico-espaciais orientadas para aptidão agrícola (AKINCl et al., 2013). Tais sistemas podem ser 
classificados como Sistemas de Suporte à Decisão Espaciais (MEYER et al., 2013). Com a possibilidade de disponibilização em rede (inclusive Internet) dos SIGs, as ferramentas elaboradas podem ser úteis a diversos usuários interessados.

Nesse contexto, defende-se ser possível a elaboração de portais computacionais em rede que conciliem SIG, SEADs de Planejamento de Uso do Solo e Bancos de Dados Conceituais. A modelagem conceitual proposta parte das bases teóricas de Vasconcelos et al. (2005) e Martins Junior et al. (2006), sob as proposições do sistema SisDec. De modo geral, conjugam-se as seguintes potencialidades:

- O Sistema de Informações Geográficas - SIG - permite ao usuário visualizar as informações sobre o território selecionado, tanto as informações primárias quanto os resultados das inferências realizadas pelos algoritmos especialistas. O SIG também serve como base de dados principal à qual os algoritmos recorrem para produzir simulações de aptidão agrícola e de planejamento territorial. A interação entre SIG e SEADs foi modelada conceitualmente na forma do Sistema SIGea (MARTINS JUNIOR et al., 2006).

- Bancos de dados conceituais podem complementar as informações de sistemas especialistas, fornecendo textos e ilustrações didáticas que auxiliem na compreensão dos conceitos e técnicas utilizados. Em alguns casos os textos podem ser disponibilizados na interface do SEAD; em outros momentos, é indicado um atalho para portais específicos de informações. A modelagem conceitual de um banco de dados conceituais que incorpore organogramas de rodas e correlações sobre Economia e Ecologia foi concebido por Martins Junior et al. (2006) e desenvolvido por Martins Junior et al. (2007), denominando-se SisOrci.

A forma de interação entre SIG, algoritmos especialistas e bancos de dados conceituais pode ser mais bem visualizada na simulação de interface apresentada na Figura 1. 


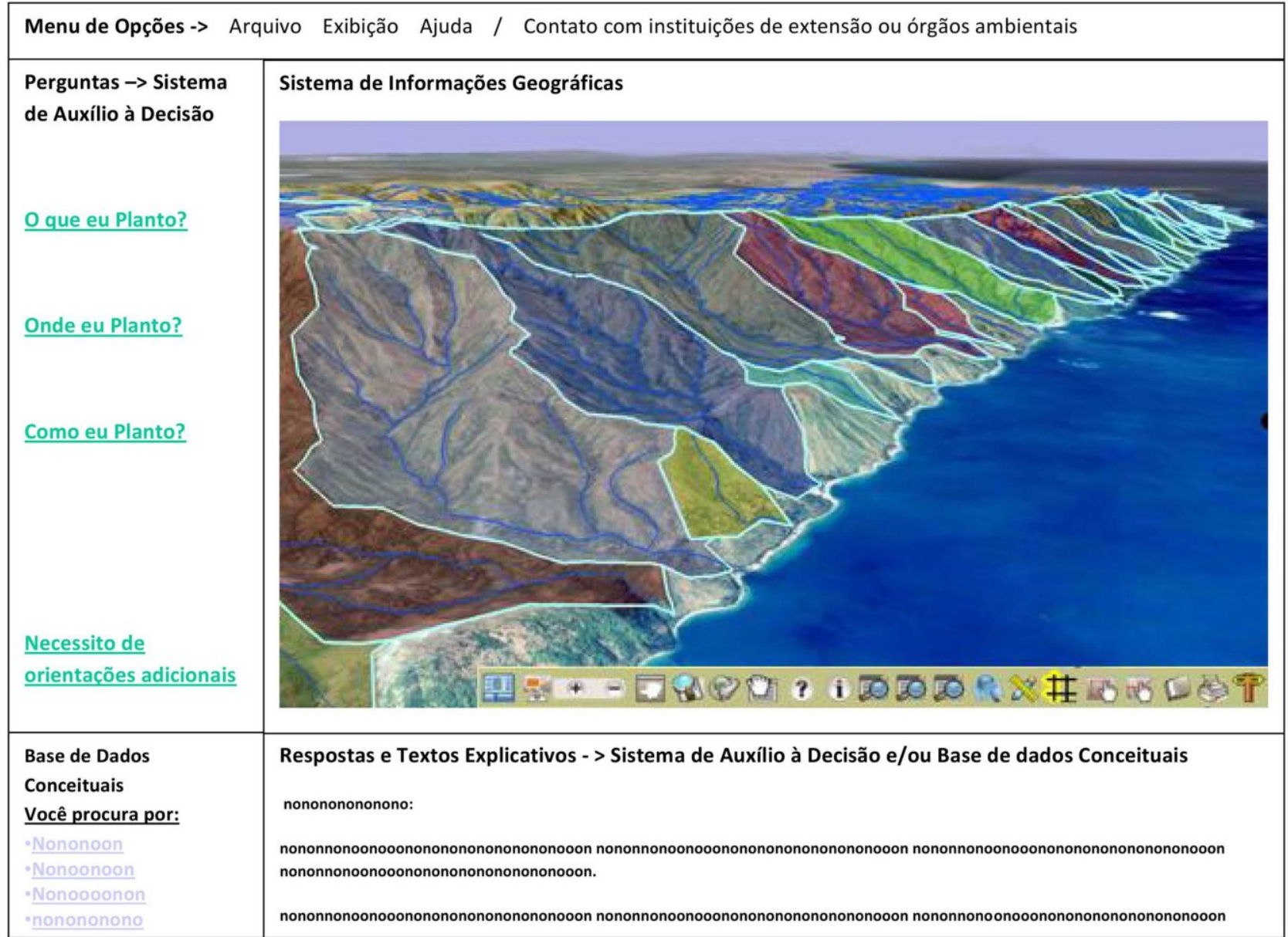

Figura 1: Simulação de Imagem de Tela da Interface do SEAD.

A identificação sobre qual o tipo de usuário (produtor rural, comitê de bacia, especialista em meio Ambiente, entre outros) permite selecionar métodos de interação diferenciados, entre o sistema de interface e o usuário. Assim, conformamse as funções disponíveis para cada perfil de usuário, e o tipo de linguagem a ser utilizado para comunicação Usuário-Programa, e as orientações adicionais úteis para cada perfil (Figura 2).

Em alguns casos, o SEAD pode interagir diretamente com o produtor rural, entregando-lhe as informações de maneira amigável e de uso direto (por exemplo, se pode plantar ou não certos cultivares, ou qual área é permitida ou proibida para plantar). Porém, muitas vezes a trabalho de análise ganhará muito mais qualidade e potenciais de aplicação quando for entregue a um intérprete ambiental ou agrônomo especialista, e daí repassado ao produtor rural. 


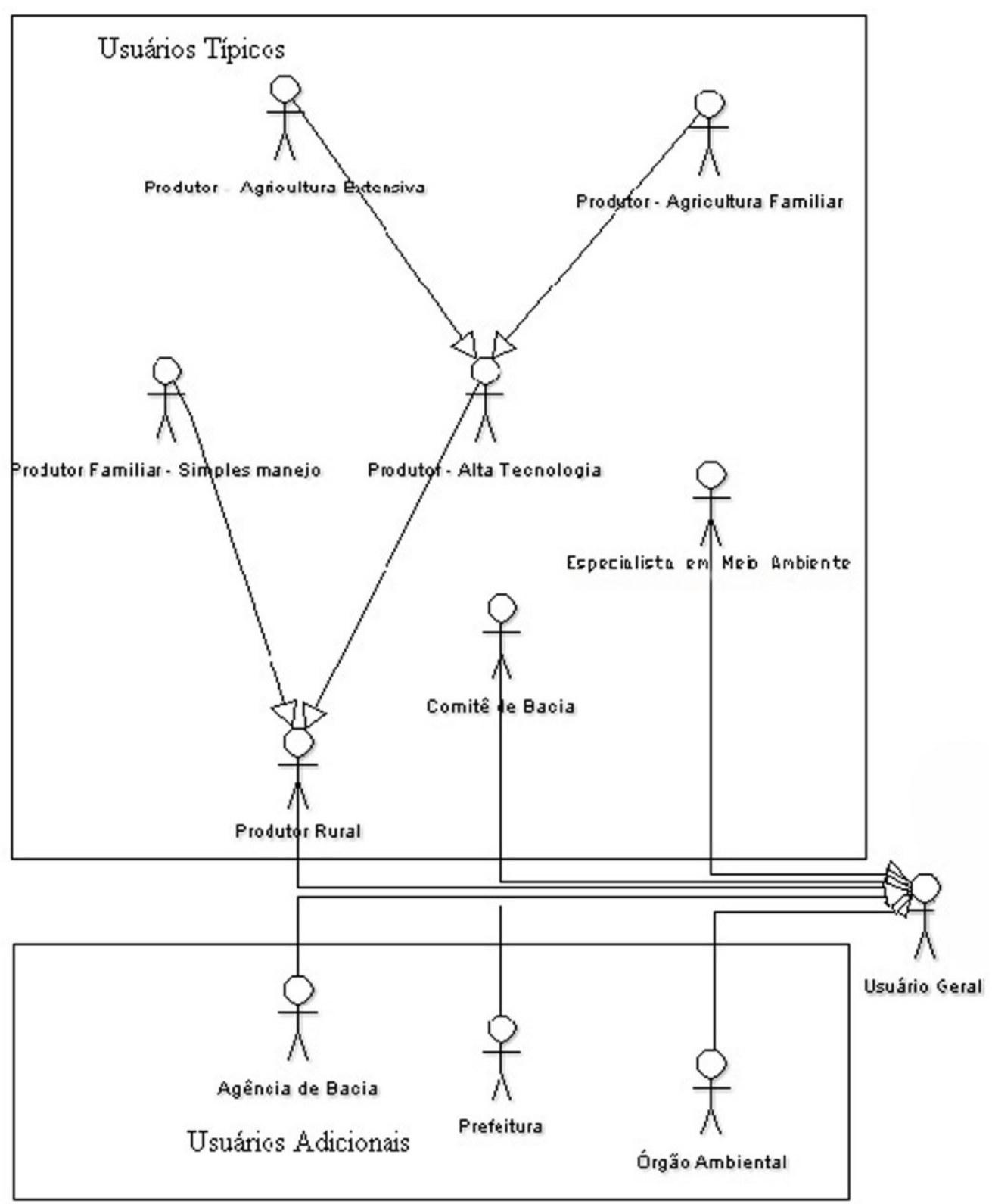

Figura 2 - Diferentes perfis de Usuários com interesse nas informações de aptidão agrícola e de planejamento de uso do solo.

Para o intérprete ambiental, não é preciso entregar apenas os produtos finais de interpretação, mas também se podem entregar os produtos intermediários, os quais serão úteis para interpretações subsequentes por parte do profissional. Isso torna úteis, no mínimo, dois níveis de resposta: uma para o usuário leigo e outra para o usuário especialista, cada uma com diferentes formatos de comunicação.

Os mapas gerados por um SEAD podem ser considerados como modelos de explicação com uso intensivo de conhecimento. Para uma melhor qualidade na comunicação de explicações de modelos de uso intensivo de conhecimento, Ginsberg (1993) aconselha que o usuário possa não apenas visualizar a resposta, mas sim obter, quando quiser, todo o caminho explicativo que levou até o resultado 
final. Esse ainda é um campo novo para os SIGs, embora seja uma preocupação tradicional na área de SEAD. Portanto, não basta saber se uma área apresenta configurações favoráveis ou desfavoráveis, mas sim saber, quando preciso, o porquê de sua rotulação nesses termos.

Portanto, é salutar que os aconselhamentos e mapas venham sempre acompanhados de uma explicação ecológica, econômica e social, relatando a justificativa para que tal resposta tenha sido escolhida. São três motivos principais para esta explicação: [1] mostrar ao usuário a confiabilidade e o embasamento das respostas oferecidas; [2] apresentar argumentos para convencê-lo a implementar o aconselhamento apresentado; e [3] atuar como instrumento de educação agropecuária e ambiental, ao tornar o usuário mais consciente das implicações de suas ações produtivas.

Entre as potencialidades de um sistema especialista que integre SIG e algoritmos de auxílio à decisão, parte-se dos seguintes potenciais de uso integrado, em sua respectiva concatenação usual:

1. Mapear o atual uso da terra.

2. Indicar as restrições Agro-Climatológicas

3. Indicar as restrições ambientais

4. Indicar as restrições legais

5. Indicar quais são as medidas de conservação necessárias, e como elas podem ser proveitosas economicamente.

6. Indicar as técnicas agrícolas adequadas (incluindo irrigação, correção de solos e adubação) para determinados cultivares.

7. Mapear o que seria o uso optimal (otimizado) da propriedade

No processo de modelagem de informações, os potenciais de uso supracitados foram integrados em três focos específicos de casos de uso: [1] A pergunta "O que eu planto?", que utiliza critérios de agroclimatologia e aptidão de solos, [2] a pergunta "Onde eu planto?", em que a primeira pergunta adquire uma dimensão espacial, e entram critérios de restrição ligados às características ambientais e hídricas sobre a área analisada, [3] a pergunta "Como eu Planto?", em que, de acordo com as informações advindas do perfil do usuário e das características da área analisada, procura-se encaminhar o usuário a um aconselhamento específico, em relação a técnicas agrícolas ecologicamente corretas e a técnicas de conservação de solo e água. 
Explicitando e sintetizando os procedimentos de modelagem de conhecimento, a seguir são apresentados os diagramas em linguagem UML, conforme preconizados pela metodologia CommonKads. No Quadro 1, apresenta-se uma estratificação dos níveis de conhecimento envolvido, em que a base apresenta os dados primários; conforme avança-se para o topo do quadro, os conhecimentos tornam-se mais abstratos e envolvem maior interação com os objetivos do usuário.

Quadro 1 - Níveis de conhecimentos envolvidos nos domínios de aptidão agrícola e planejamento de uso da terra.

\begin{tabular}{|c|c|}
\hline $\begin{array}{l}\text { CONHECIMENTO DE } \\
\text { TAREFA }\end{array}$ & $\begin{array}{l}\text { Raciocínio das perguntas: } \\
\text { - O que eu planto? } \\
\text { - Onde eu planto? } \\
\text { - Como eu planto? }\end{array}$ \\
\hline $\begin{array}{l}\text { CONHECIMENTO DE } \\
\text { INFERÊNCIA }\end{array}$ & $\begin{array}{l}\text { Inferências Básicas sobre o domínio: } \\
\text { - Classificação (por aptidão de cultivar e técnica) } \\
\text { - } \quad \text { Diagnóstico (para aconselhamento) }\end{array}$ \\
\hline \multirow{3}{*}{$\begin{array}{l}\text { CONHECIMENTO DE } \\
\text { DOMÍNIO }\end{array}$} & $\begin{array}{l}\text { Comportamento Aconselhável no Ambiente } \\
\text { - Limites de Tolerância Ambiental } \\
\text { - Técnicas Corretas de Manejo }\end{array}$ \\
\hline & Regras, Relações e Processos Aplicáveis ao Ambiente \\
\hline & Atributos do Ambiente \\
\hline
\end{tabular}

A Figura 3, por sua vez, apresenta variáveis básicas a serem manipuladas na base de dados. Não se pretende esgotar as possibilidades de variáveis a serem utilizadas, mas apenas demonstrar a natureza dessas e o relacionamento básico entre os grupos de variáveis.

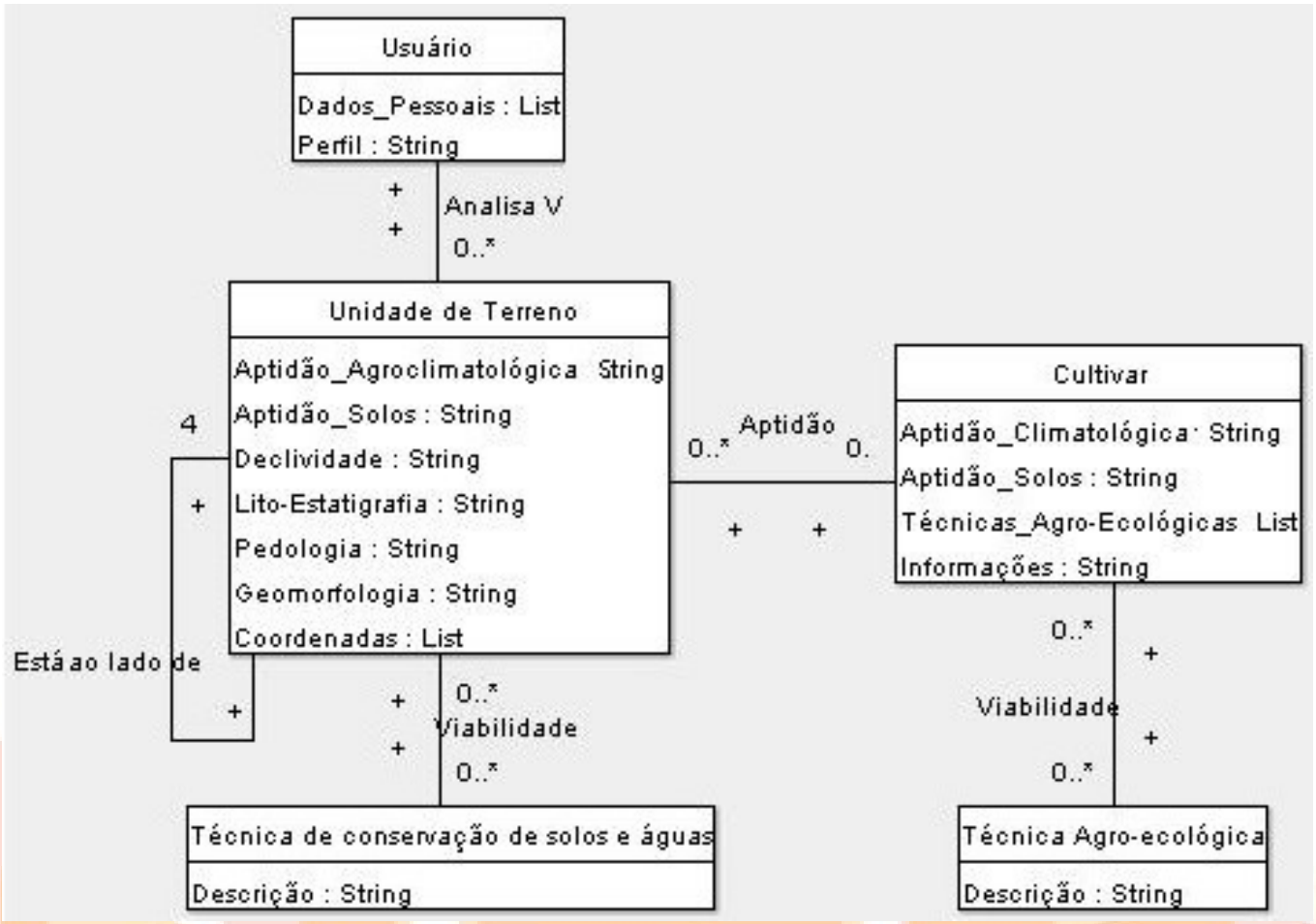

Figura 3 - Diagrama de Classes, detalhando os objetos e variáveis a serem manipuladas por SEAD. 
A Figura 4, conseguinte, apresenta a rotina básica de funcionamento do SEAD. Após a definição do perfil de usuário, as funcionalidades podem ser acessadas paralelamente, a partir de interface como a apresentada na Figura 1.

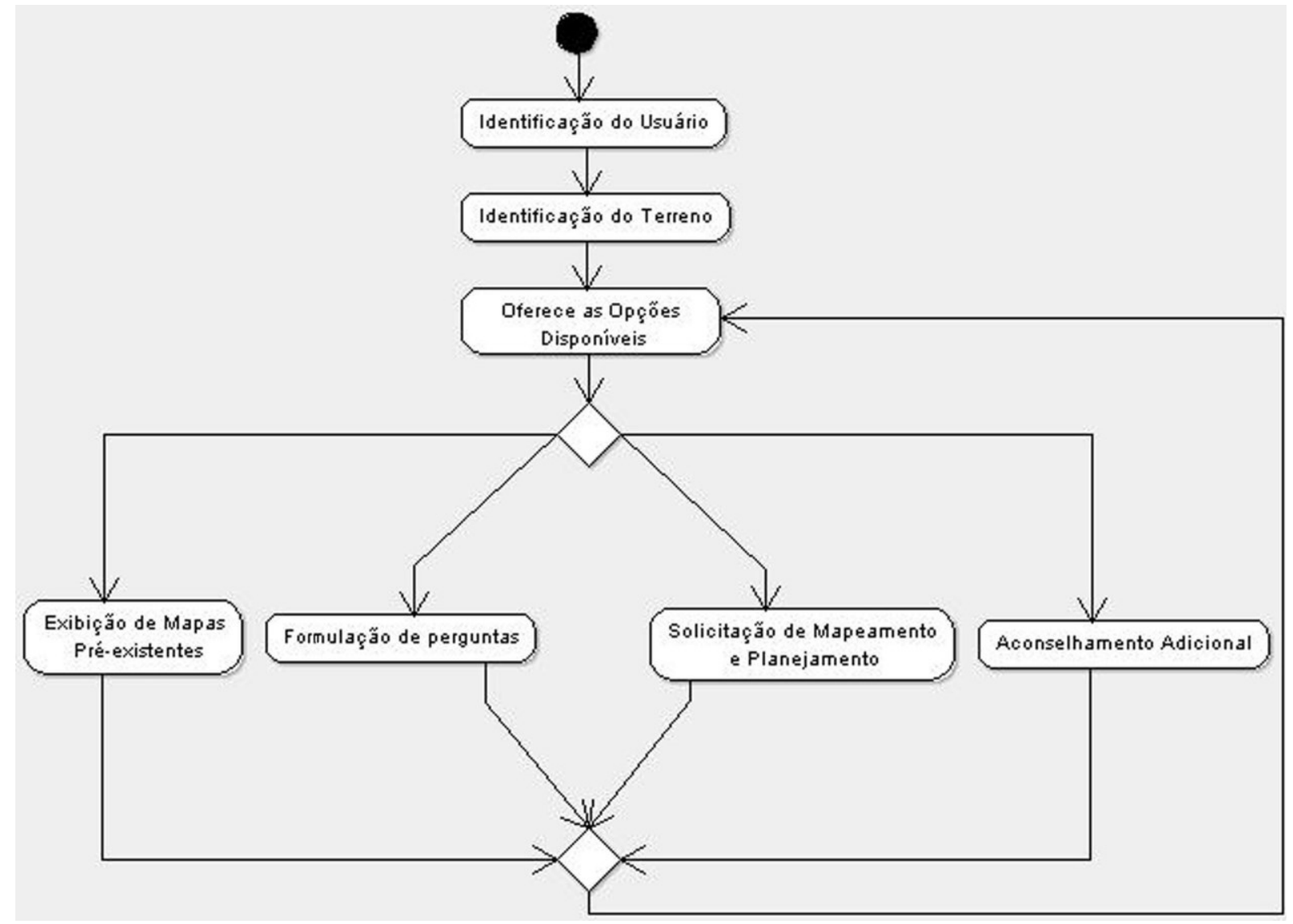

Figura 4- Diagrama de Atividades descrevendo os modos de interação entre Usuário e o sistema especialista no decorrer de uma execução típica.

Os diagramas de atividade das Figuras 5, 6 e 7 detalham os fluxos de procedimentos envolvidos em cada caso de uso ( $\mathrm{O}$ quê, onde e como planto). Cabe ressaltar que pode ser possibilitado ao usuário inserir novas informações (cartográficas ou pontuais) que auxiliem no processo de auxílio à decisão. Essas variáveis inseridas, a critério, podem inclusive ser compartilhadas em rede, de forma a aprimorar a estrutura de informações sobre o território. A inserção de informações também é uma das características principais para flexibilizar o sistema ao trabalho em múltiplas escalas.

A análise interescalar traz à tona a hipótese de que abordagens em diferentes escalas podem necessitar de diferentes metodologias de aferição de aptidão agrícola e planejamento de uso da terra. Para as escalas de maior abrangência territorial, existem metodologias consolidadas, como as de zoneamento agroclimatológicos (por cultivar) (LUPPI et al., 2014) e as metodologias de aptidão 
geral, tais como Avaliação de Capacidade de Uso do Solo (LEPSCH et al., 1991), e o Sistema FAO/Brasileiro de Avaliação de Aptidão Agrícola das Terras (RAMALHO FILHO e BEEK, 1995; aprimorado por PEREIRA e LOMBARDI NETO, 2004 e MARQUES et al., 2012). O uso complementar dos mapas de aptidão geral e dos mapas de zoneamento agroclimatológico por cultivares permite uma primeira aproximação do desenho de uso produtivo optimal (otimizado) de um território (MARTINS JUNIOR et al., 2006).

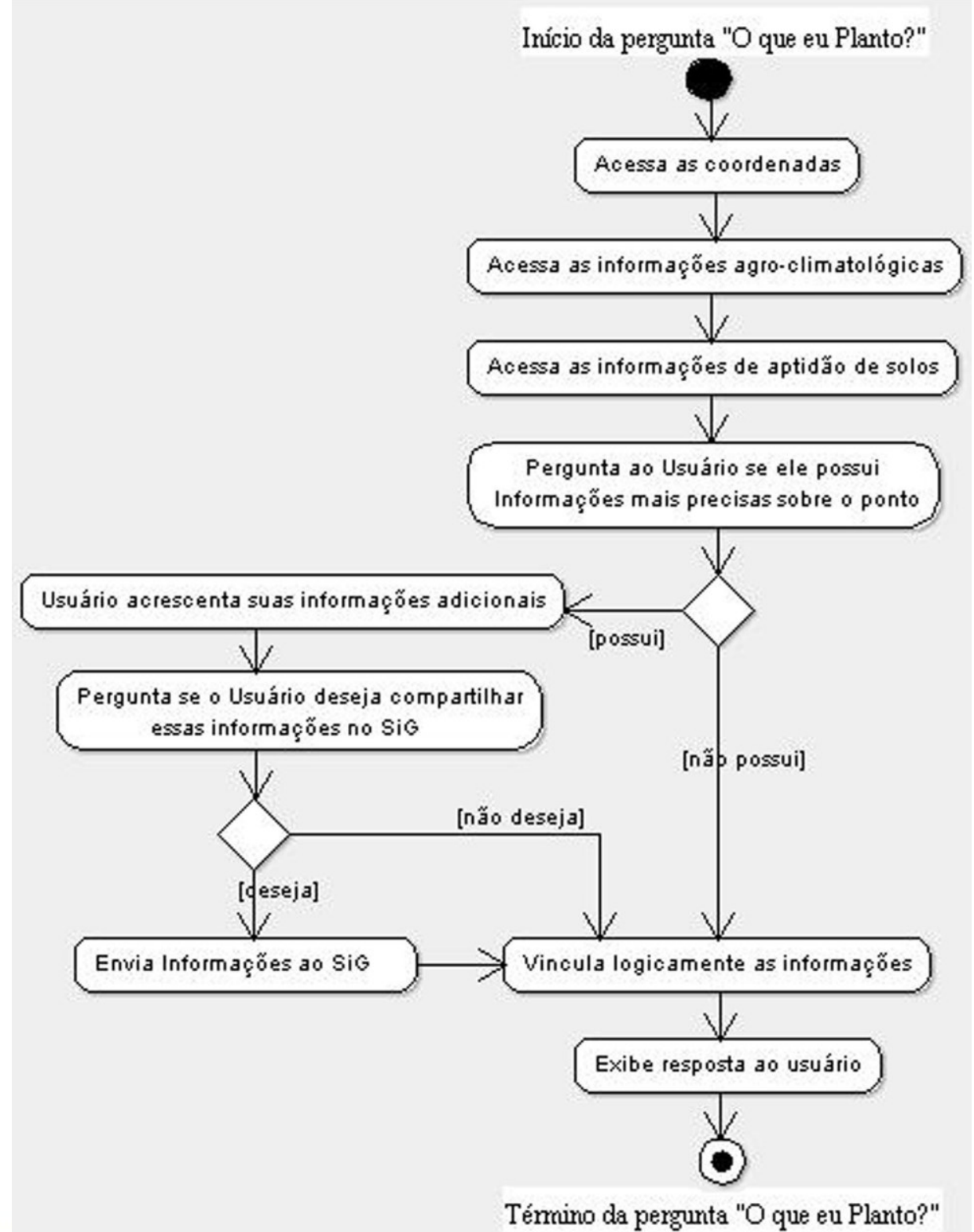

Figura 5 - Diagrama de Atividades detalhando a modelagem lógica inicial do raciocínio explícito abarcado pela questão "O que eu Planto?".

Entretanto, quando se passa a trabalhar em microescala (1:10.000 ou menos, ou seja, discriminando áreas dentro de uma propriedade rural), desponta a necessidade de considerar uma grande diversidade de outras variáveis, entre as 
quais: microclima; profundidade do solo; balanço de nutrientes; profundidade e variação de lençol freático; matéria orgânica; entre vários outros. Pode-se proferir que, ao se transitar da macro para a microescala (por exemplo, de um mapa de um Estado ou município para um mapa de uma propriedade rural), a utilidade das generalizações perde espaço para a complexidade da realidade de campo. A habilidade em lidar com os diversos atributos locais visando a um sistema produtivo eficiente depende em grande parte da experiência tácita do produtor e do extensionista. Como experiência tácita, entende-se aquela adquirida pela vivência prática, sendo não-codificada ou não-estruturada (PORTO, 2011). Nesses termos, caberia o questionamento sobre se existe a possibilidade de ser possível traduzir esse conhecimento em uma estrutura lógica formalizada de uma base computacional. 


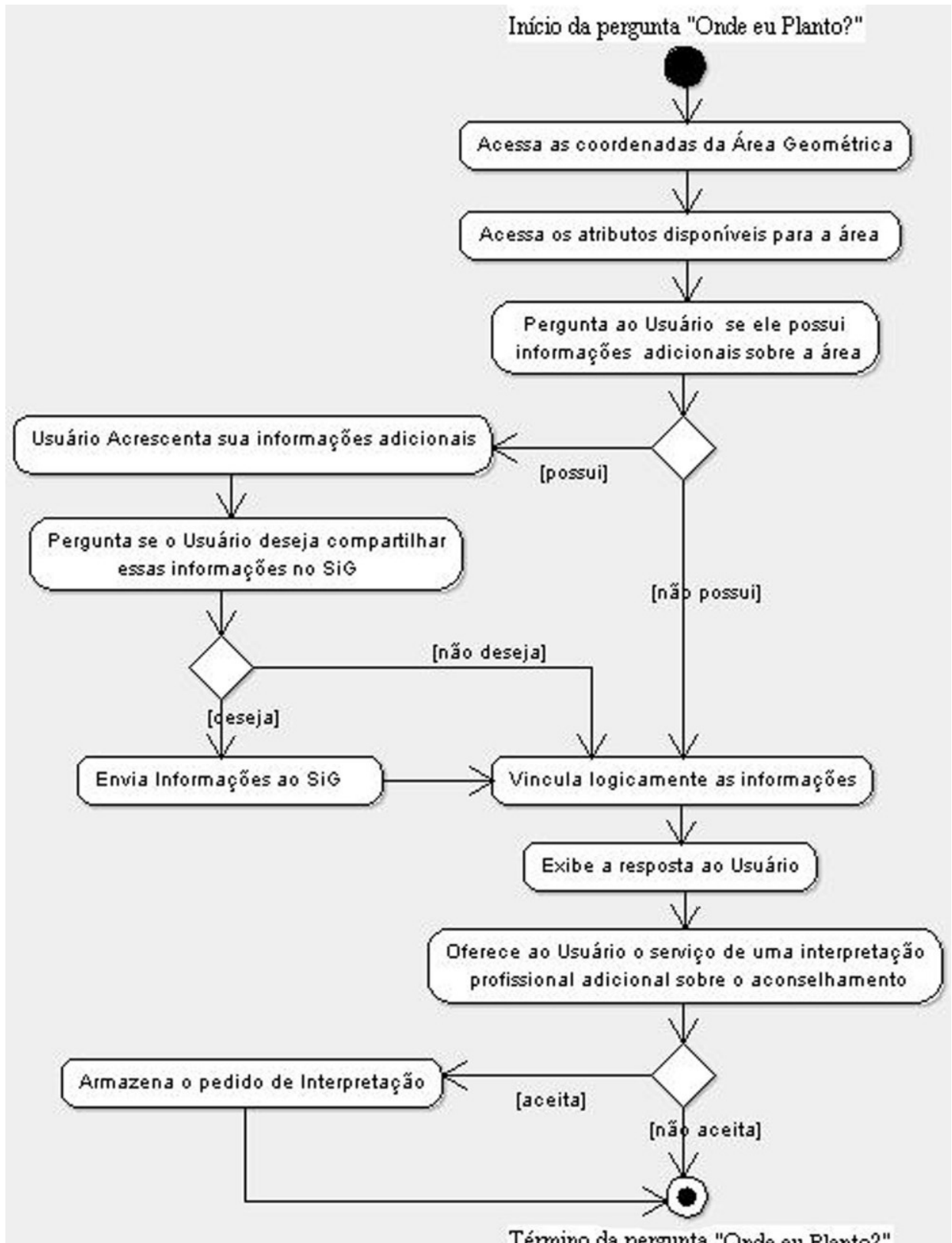

Figura 6 - Diagrama de Atividades detalhando a modelagem lógica inicial do raciocínio explícito abarcado pela questão "Onde eu Planto?".

R. Inter. Interdisc. INTERthesis, Florianópolis, v.12, n.2, p.221-245, Jul-Dez. 2015 


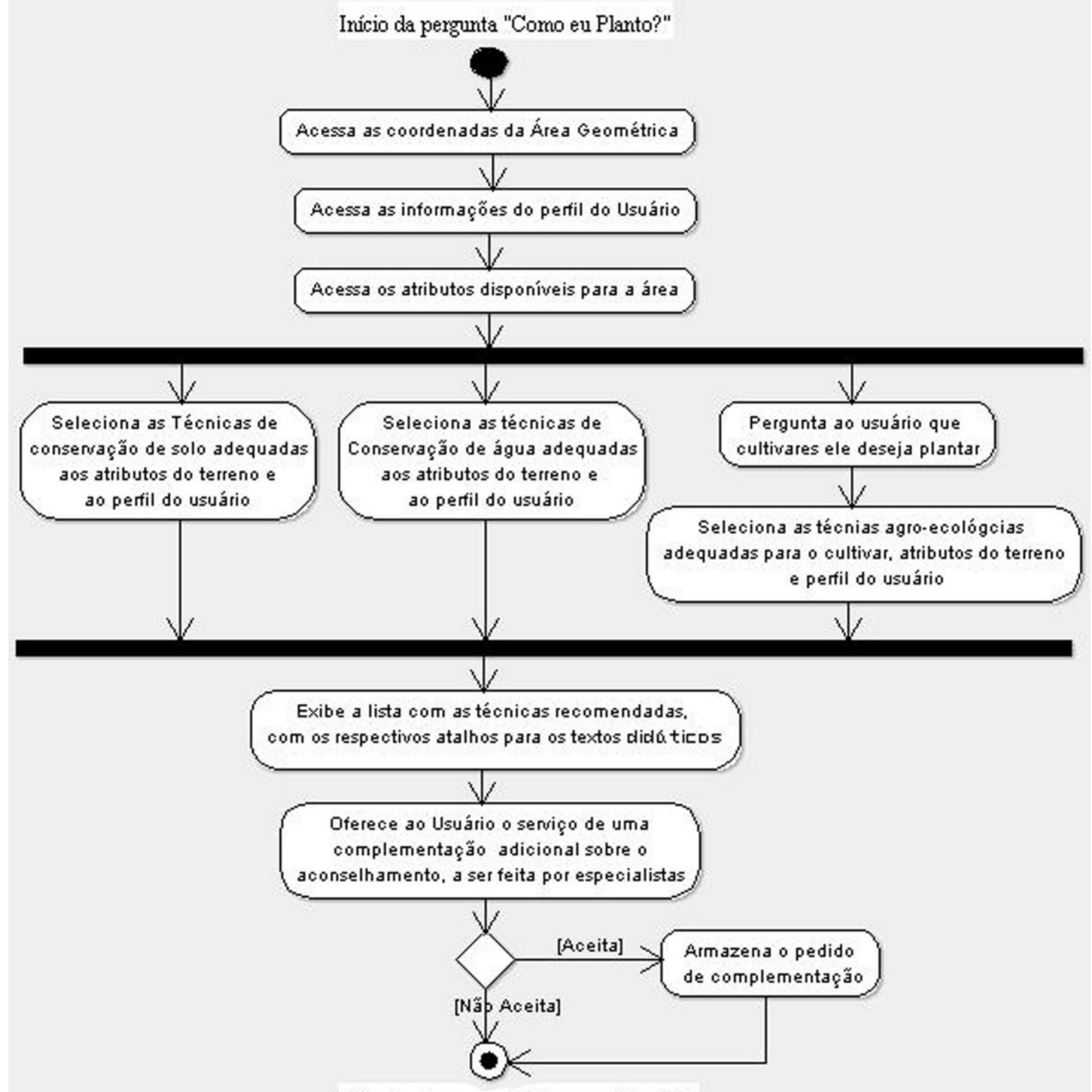

Término da pergunta "Como eu Planto?"

Figura 7 - Diagrama de Atividades detalhando a modelagem lógica inicial do raciocínio explícito abarcado pela questão "Como eu Planto?".

De forma a confrontar esse desafio epistemológico, elaborou-se uma pequena sistematização de critérios de aptidão agrícola a partir das seguintes obras de compilação de aconselhamentos agronômicos: Lainetti e Brito (1986) e de Queiroz et al., (1980). Procurou-se identificar os atributos mais recorrentes entre os vários cultivares, bem como determinar o efeito do atributo ambiental sobre a aptidão agrícola de cada espécie. A sistematização das informações seguiu o método das matrizes lógicas interdisciplinares (MARTINS JUNIOR et al., 2006), e é apresentado no Quadro 2.

Ressalta-se que as obras de Lainetti e Brito (1986) e de Queiroz et al. (1980) são apenas compilações generalistas preliminares - uma abordagem científica mais 
detalhada para aptidão agrícola em micro-escala necessitaria de um recolhimento de publicações técnicas específicas para cada cultivar. Portanto, longe de esgotar o tema, o Quadro 2 apenas aponta a viabilidade de se construir uma estrutura informacional que unifique os dados de aptidão agrícola que hoje encontram-se dispersos em diversos estudos e boletins agronômicos. A integração dos dados de aptidão de cada cultivar permitiria comparações computacionais entre quais seriam os cultivares mais adequados para produção de acordo com as características do terreno em simulações de microescala.

Quer durante a modelagem dos conhecimentos, que em seu ciclo de avaliação e aprimoramento, as seguintes questões que devem ser mantidas em consideração quanto à eficácia do auxílio à decisão, nas várias escalas de abordagem e perfis de usuário (VASCONCELOS et al. 2005, p.14): [1] o que é legítimo de fazer em dada circunstância no interesse econômico, em consistência com o aspecto ambiental [2] em que medida o impacto ambiental a ser gerado deve ser tolerado, i.e., em casos extremos como de agricultura extensiva, com qual limite ao se procurar cobrir grandes áreas com projetos executivos de agricultura [3] quais são as alternativas face aos interesses agrícolas diante das restrições ambientais [4] o que pode ser remediado por soluções técnicas mitigadoras e preventivas, e a quais custos para que o enfoque econômico-ecológico seja respeitado e [5] o que é proibido legalmente.

Para tentar aferir sua eficácia, é possível a disponibilização de um sistema de feedback (retroalimentação de informações), onde o usuário poderá escrever se concorda ou não com o aconselhamento, e o porquê. Outra forma de interação é incluir outras perguntas ao usuário, procurando saber se ele pretende empregar ou não o conselho, e caso a resposta seja negativa, indagar o motivo.

Uma terceira via complementar é fornecer ao usuário um espaço para relatar se o conselho dado teve sucesso ou não, quando executado. Esse mecanismo de feedback torna-se muito importante para os futuros melhoramentos do sistema. Em uma perspectiva mais ampliada, a retroalimentação de informações permite inclusive avaliar a eficácia das metodologias de aptidão agrícola e planejamento de uso da terra utilizadas. 
Quadro 2 - Matriz Interdisciplinar explicitando a relação de Aptidão Agrícola por Cultivar com Atributos de Solo e Relevo

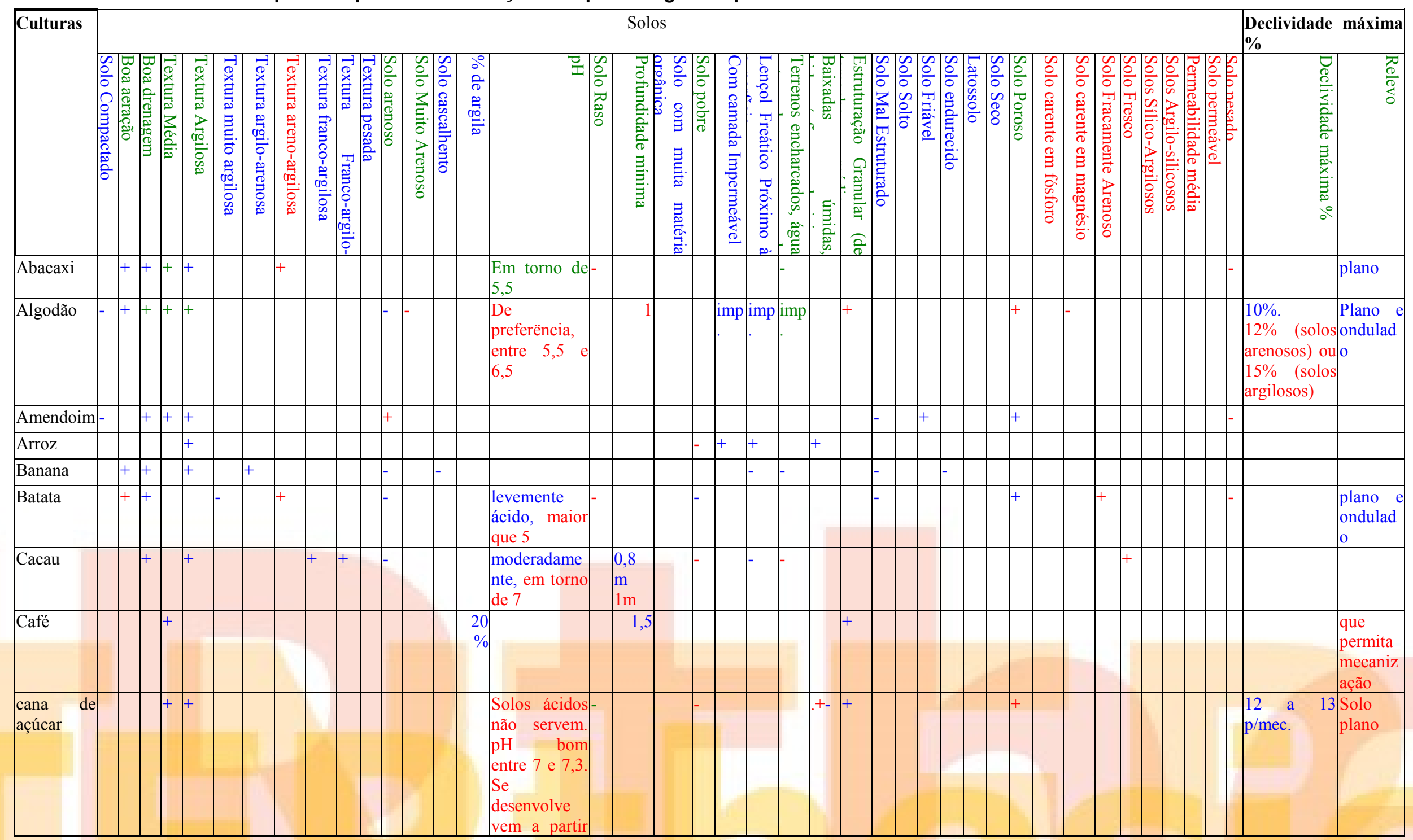

R. Inter. Interdisc. INTERthesis, Florianópolis, v.12, n.2, p.221-245, Jul-Dez. 2015 


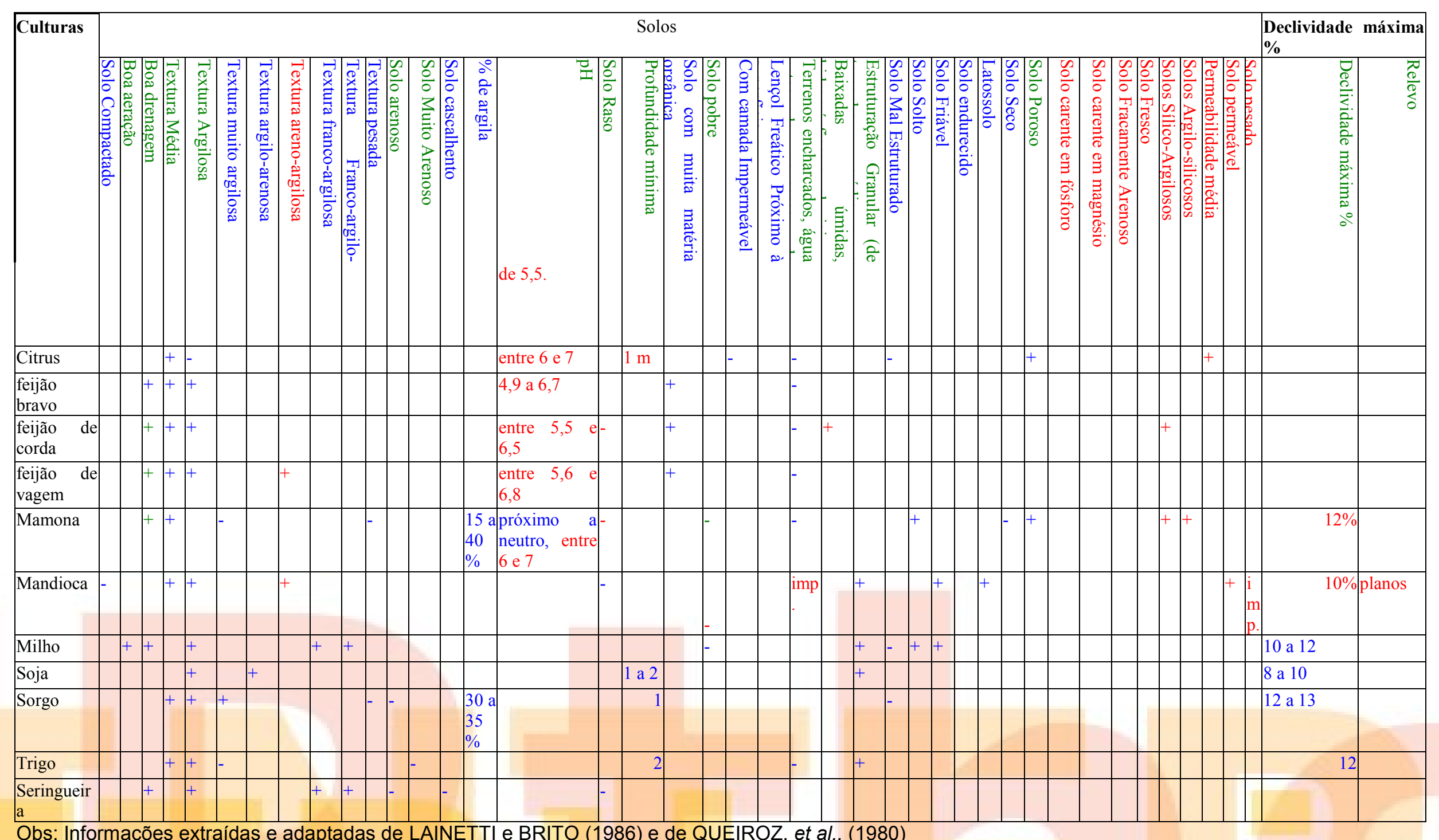

Obs: Informações extraídas e adaptadas de LAINETTI e BRITO (1986) e de QUEIROZ, et al., (1980)

R. Inter. Interdisc. INTERthesis, Florianópolis, v.12, n.2, p.221-245, Jul-Dez. 2015 
Legenda:

+ Característica propícia ao cultivar

- Característica restritiva ao cultivar

Atlas agroclimatológico (QUEIROZ et al., 1980)

Guia de culturas de A a Z (LAINETTI e BRITO, 1986)

Ambas as fontes

\section{CONCLUSÕES}

Os procedimentos de modelagem do conhecimento mostraram ser possível desenvolver uma estrutura formal de informações e algoritmos para auxiliar na interpretação de aptidão agrícola e planejamento territorial, em múltiplas escalas. Projetos piloto de SEADs já têm sido testados para a seleção agroclimatológica de cultivares ("O que plantar?) (HARTATI e SITANGGANG, 2010); zoneamentos genéricos de aptidão agrícola ("Onde Plantar") em macroescala (MOURA, 2007) e microescala (DELARMELINDA et al., 2014); e procedimentos de manejo agrícola ("Como Plantar) (KARMAKAR et al., 2007). Não obstante, a estrutura de informações proposta neste artigo acena a utilidade de se integrar as informações, técnicas e metodologias de aptidão agrícola nas três abordagens apresentadas, em uma arquitetura de conhecimentos ainda mais ampliada.

O principal benefício do SEAD modelado é oferecer auxílio à decisão em planejamento territorial para agricultores e gestores de bacia hidrográfica. As informações de aconselhamento podem ser úteis tanto sobre uma região onde se está para iniciar uma atividade produtiva na propriedade, quanto para uma região que já possua uma ocupação efetiva do território (BAJA et al., 2001).

O referido SEAD, sob uma interpretação ampla, fornece em um conjunto de injunções lógicas geoambientais que permitem escolher e optar por diversas soluções de uso do território com vistas à manutenção da estabilidade geral dos sistemas naturais envolvidos, produzir um mínimo de irreversibilidade em qualquer dos subsistemas, garantido o objetivo adequado de produtividade econômica (PEREIRA, 2002, p. 13-17; MARTINS JUNIOR et al., 2005; PEREIRA et al., 2006). As consequências lógicas a partir dos atributos espaciais geram as recomendações disponibilizadas aos usuários.

Concomitantemente, o sistema proposto afigura-se um eficaz instrumento de educação agropecuária e ambiental, por propor alternativas ecologicamente sustentáveis ao usuário e explicar a justificativa econômica para tais propostas. 
Dessa maneira, tenciona-se que os usuários passem a assumir uma postura mais coerente em relação as suas atividades de uso territorial, incorporando em seu escopo de decisão as corretas variáveis ambientais, com suas implicações na produtividade econômica. Ressalta-se que tal sistema permite criar um eixo de articulação entre produtores rurais, gestores de bacia hidrográfica e gestores ambientais, por integrar os três modos de planejamento territorial em uma visão de raciocínio ecológico-econômica, como preconizado por Pereira et al. (2006).

Apesar de sua patente utilidade para auxiliar um planejamento sustentável do uso da terra, os SEAD modelados neste artigo, apresentam as seguintes limitações:

- Dificuldades de modelagem do conhecimento tácito agronômico e na realização de generalizações a partir destas;

- Necessidade de informações especializadas em escala de detalhe para fornecer a orientação adequada;

- Limitações na capacidade técnica dos usuários para inserir informações, compreender as orientações e aplicá-las efetivamente;

- Dificuldades de acesso a computadores e à Internet no meio rural.

Todavia, com o progressivo desenvolvimento tecnológico e socioeconômico, espera-se que essas limitações sejam minoradas gradualmente e que os SEAD possam se tornar de uso mais corrente em um futuro próximo. 
AGRADECIMENTOS

Agradecemos ao CNPq, FINEP, CAPES e FAPEMIG pelo financiamento das pesquisas que possibilitaram a elaboração deste artigo. 


\title{
INFORMATION SCIENCE AND AGRICULTURAL SUITABILITY: INTER-SCALE APPROACHES FOR LAND USE PLANNING
}

\begin{abstract}
:
Technical and methodological innovations in programming, network sharing, information system analysis and geographic information systems (GIS) have enabled the representation, storage, processing and dissemination of information that was previously unavailable to interested parties. In this context, this study presents a proposal for the conceptual modeling of the integrated use of agricultural land suitability methods to develop support systems for land use decision-making information science methods. Knowledge-based modeling diagrams are structured using CommonKADS and Unified Modeling Language (UML) methods to simulate the processing of basic questions from farmers, including "what to plant", "where to plant", and "how to plant". Finally, an interdisciplinary matrix, which explains the relationship between crop-specific agricultural land suitability and soil and terrain attributes to support decision-making at a detailed scale, is presented. The proposed system is an effective instrument for agricultural and environmental guidance and education because it provides environmentally sustainable alternatives to the user and explains the economic rationale for such proposals.
\end{abstract}

Keywords: Agronomy. Geographic Information Systems. Information Science. Decision-Making Support Systems. Environment.

\section{CIÊNCIA DE LA INFORMACIÓN Y APTITUD AGRÍCOLA: ENFOQUES INTER- ESCALARES PARA PLANIFICACIÓN DEL USO DE LA TIERRA}

\section{Resumen:}

Las innovaciones técnicas e metodológicas de programación, compartición en red, análisis de sistemas de informaciones e sistemas de información geográfica (SIG) permiten la representación, almacenamiento, tratamiento y diseminación de informaciones que anteriormente no estaban disponibles para los diversos actores interesados. A partir de este contexto, se presenta una propuesta de modelización conceptual acerca del uso integrado de las metodologías de aptitud agrícola para la implementación de sistemas de soporte a la decisión acerca del uso del suelo, por intermedio de metodologías de la Ciencia de la Información. Se estructuran diagramas de modelaje del conocimiento por los métodos CommonKADS y Unified Modeling Language (UML) para simular el tratamiento de cuestiones básicas de un productor rural, tales como “¿Qué puedo plantar?”, “¿Dónde plantar?”, “¿Cómo plantar?" Por último, una matriz interdisciplinaria es presentada, clarificando las relaciones entre aptitud agrícola con atributos del suelo y del relieve, de modo a auxiliar en la tomada de decisiones en escala de detalles. Lo sistema propuesto puede ser una instrumento eficaz para la orientación y educación agrícola y ambiental, por demonstrar alternativas ecológicamente sostenibles, mientras explana las justificación económica para tales alternativas.

Palabras clave: Agronomía, Sistemas de Información Geográfica, Ciencia de la Información, Sistemas de Soporte a la Decisión, Medio Ambiente 


\section{REFERÊNCIAS}

AKINCI, H.; ÖZALP, A. Y.; TURGUT, B. Agricultural land use suitability analysis using GIS and AHP technique. Computers and electronics in agriculture, 97, 7182. 2013.

ASSAD, E. D., MARTINS, S. C., BELTRÃO, N. E.M.; PINTO, H. S. Impactos das mudanças climáticas no zoneamento agrícola de risco climático para o cultivo de algodão no Brasil. Pesquisa Agropecuária Brasileira, 48(1), 1-8. 2013.

BAJA, S., CHAPMAN, D.M., DRAGOVICH, D. A conceptual model for assessing agricultural land suitability at a catchment level using a continuous approach in GIS. Geospatial Information and Agriculture, The Regional Institute Ltd. 2001.

BOLFE, É. L., VICENTE, L. E., SPINELLI-ARAÚJO, L., VICTORIA, D.C, TÔSTO, S. G., TORRESAN, F., QUARTAROLI, C.F.; FURTADO, A.L.S.; MARTINS, M. A. M. Banco de Dados Geoespaciais do Macrozoneamento Ecológico-Econômico do Estado do Maranhão. In: SIMPÓSIO BRASILEIRO DE SENSORIAMENTO REMOTO, 17., 2015, João Pessoa. Anais... São José dos Campos: INPE, 2015.

DELARMELINDA, E. A., WADT, P. G. S., ANJOS, L. H. C., MASUTTI, C. S. M., SILVA, Ê. F., SILVA, M. B., COELHO, R.M.; SILVA, L.M.; SHIMIZU, S.H.; COUTO, W. H. Aplicação de sistemas de avaliação da aptidão agrícola das terras em solos do Estado do Acre, Amazônia. Biota Amazônia, 4(2): 87-95. 2014.

FAISAL, C.M.N.; AWAN, M.S.A.; ARSHAD, M.S. Knowledge Management: A Particle Approach to Design Knowledge based Portal Using CommonKADS. Lap Lambert, 2011.60p.

GINSBERG, Matt - Essentials of Artificial Inteligence -Morgan Kaufmann Publihsers - USA, 1993.

HARTATI, S.; SITANGGANG, I. S. A fuzzy based decision support system for evaluating land suitability and selecting crops. Journal of Computer Sciences. 6(4):417-424, 2010

KARMAKAR, S.; LAGUË, C.; AGNEW, J.; LANDRY, H. Integrated decision support system (DSS) for manure management: A review and perspective. Computers and Electronics in Agriculture, 57(2), 190-201. 2007. 
LAINETTI, R., BRITO, NRS. As Culturas de A até Z. Guia Rural Abril, Ed. Abril, São Paulo, 1986.

LEPSCH, I. F.; BELLINAZZI JR., R.; BERTOLINI, D.; ESPÍNDOLA, Carlos Roberto. Manual para levantamento utilitário do meio físico e classificação de terras no sistema de capacidade de uso. 4a aproximação. Campinas: SBCS, 1991. 175p.

LUPPI, A.S.L.; SANTOS, A.R.; EUGÊNIO, F.C.; BRAGANÇA, R.; PELUZIO, J.B.E.; DALFI, R.L. SILVA, R.G. Metodologia para classificação de zoneamento agroclimatológico. Revista Brasileira de Climatologia. 10 (15): 80-97. Jul/Dez 2014

MANZATTO, C. V.; ASSAD, E. D.; BACCA, J. F. M.; ZARONI, M. J.; PEREIRA, S. E. $\mathrm{M}$. Zoneamento agroecológico da cana-de-açúcar. Rio de Janeiro: Embrapa Solos. 2009

MARQUES, F.S.M.; MARTINS JUNIOR, P.P.; VASCONCELOS, V.V.; NOVAES, L.A.d`A. Proposição Metodológica para a Cartografia de Solos e Aptidão Agrícola: Estudo de Caso para a Bacia do Rio Paracatu. Revista Brasileira de Geografia Física, Vol. 5, No 1 (2012) 01-17.

MARTINS JUNIOR, P.P.; ROSA, S.A.G., CANTISANO, M.A.M., GOMES, L.A. M.. Zoneamentos Econômicos de Bacias Hidrográficas - Importância Ecológica. Revista ECEN - Economia \& Energia, Ano XII. No 71, Dezembro, 2008 - Janeiro, 2009.

MARTINS JUNIOR, P.P. et al. Projeto ACEE - Arquitetura de Conhecimentos em Ecologia-Economia. MCT/CNPq 2005-2007. Relatório Final em 2007.

MARTINS JUNIOR, P.P. et al.. Projeto CRHA - Conservação de Recursos Hídricos no âmbito de Gestão Agrícola de Bacias Hidrográficas.

MCT/FINEP/CT-HIDRO 2002-2006. Relatório Final em 2006.

MARTINS JUNIOR, P.P. et al. Projeto SACD - Sistemas de Arquitetura de Conhecimentos e de Auxílio à Decisão na Gestão Geo-Ambiental e Econômica de Bacias Hidrográficas e Propriedades Rurais. Fapemig (2010-2011). Relatório Final em 2012.

MEYER, A., ESTRELLA, R., JACXSENS, P., DECKERS, J., VAN ROMPAEY, A.,; VAN ORSHOVEN, J. A conceptual framework and its software implementation to generate spatial decision support systems for land use planning. Land Use Policy, 35, 271-282. 2013. 
MOURA, L. C. (2007). A ocupação espaçotemporal dos cafezais no município de Machado, no Sul de Minas: a relação entre aptidão agrícola da terra e seu uso na atividade cafeeira. Tese (Doutorado). Universidade Federal de Lavras. Lavras-MG. $117 p$.

OZAKI, V. A. O papel do seguro na gestão do risco agrícola e os empecilhos para o seu desenvolvimento. Revista Brasileira de Risco e Seguro, 2(4), 75-92. 2007.

PEREIRA, L. C. Aptidão Agrícola das terras e sensibilidade ambiental: proposta metodológica. Tese de Doutorado. Faculdade de Engenharia Agrícola. Universidade Estadual de Campinas (SP). 2002. 135p.

PEREIRA, L.C.; SILVEIRA, M.A.; LOBARDI NETO, F. Agroecologia e Aptidão de Terras: as bases científicas para uma agricultura sustentável. Embrapa Meio Ambiente: Jaguariuna, 2006, 5p.

PEREIRA, L.C.; LOMBARDI NETO, F. Avaliação da aptidão agrícola das terras: proposta metodológica. Jaguariúna: Embrapa Meio Ambiente, Documentos, $n^{\circ} 43$. 2004. ISSN 1516-4691

PORTO, Rodrigo Amorim. Gestão do conhecimento: os desafios de explicitar conhecimentos técnicos. 2011. 55 f., il. Monografia (Bacharelado em Administração)—Universidade de Brasília, Brasília, 2011.

QUEIROZ, R., SOUZA, A.G., SANTANA, D.P., ANTUNES, F.Z, FONTES, M. (coord.) - Zoneamento Agroclimatológico de Minas Gerais - Governo de Minas Gerais, Secretaria de Agricultura, Belo Horizonte, 1980.

RAMALHO FILHO, A.; BEEK, K. J. Sistema de avaliação da aptidão agrícola das terras. 3.ed. Rio de Janeiro: EMBRAPA-CNPS, 1995. 65p.

SCHNEIDER, L. M., ROLIM, G. D. S., SOBIERAJSKI, G. D. R., PRELA-PANTANO, A.; PERDONÁ, M. J. Zoneamento agroclimático de nogueira-macadâmia para o Brasil. Revista Brasileira de Fruticultura, 34, 515-524. 2012.

SEIDL, M., SCHOLZ, M., HUEMER, C.; KAPPEL, G.. UML@ Classroom: An Introduction to Object-Oriented Modeling. Springer. 2015. 206 pages

VASCONCELOS, V.V, MARTINS JUNIOR, P.P., NOVAES, L. A. d'A. Sistema 
Inteligente de Apoio à Decisão em Gestão de Recursos Hídricos e Projetos Agrícolas - XVI SIMPÓSIO BRASILEIRO DE RECURSOS HÍDRICOS, Anais... 2005. 20 p.

WOLLMANN, C. A.; GALVANI, E.. Zoneamento agroclimático: linhas de pesquisa e caracterização teórica-conceitual. Revista Sociedade \& Natureza, Uberlândia, 25(1). 2013.

WOLLMANN, C. A.; GALVANI, E. Zoneamento Agroclimático. Buqui Livros Digitais. 2014. 149p.

Artigo:

Recebido em 04 de Junho de 2015

Aceito em 11 de Novembro de 2015 